\title{
Modelling the dynamics of Plasmodium falciparum histidine-rich protein 2 in human malaria to better understand malaria rapid diagnostic test performance
}

\author{
Louise Marquart', Alice Butterworth ${ }^{2,3}$, James S McCarthy ${ }^{2,3}$ and Michelle L Gatton ${ }^{4 *}$
}

\begin{abstract}
Background: Effective diagnosis of malaria is a major component of case management. Rapid diagnostic tests (RDTs) based on Plasmodium falciparumhistidine-rich protein 2 (PfHRP2) are popular for diagnosis of this most virulent malaria infection. However, concerns have been raised about the longevity of the PfHRP2 antigenaemia following curative treatment in endemic regions.
\end{abstract}

Methods: A model of PfHRP2 production and decay was developed to mimic the kinetics of PfHRP2 antigenaemia during infections. Data from two human infection studies was used to fit the model, and to investigate PfHRP2 kinetics. Four malaria RDTs were assessed in the laboratory to determine the minimum detectable concentration of PAHRP2.

Results: Fitting of the PfHRP2 dynamics model indicated that in malaria naive hosts, P. falciparum parasites of the 3D7 strain produce $1.4 \times 10^{-13} \mathrm{~g}$ of PAHRP2 per parasite per replication cycle. The four RDTs had minimum detection thresholds between 6.9 and $27.8 \mathrm{ng} / \mathrm{mL}$. Combining these detection thresholds with the kinetics of PFHRP2, it is predicted that as few as 8 parasites/ $\mu \mathrm{L}$ may be required to maintain a positive RDT in a chronic infection.

Conclusions: The results of the model indicate that good quality PAHRP2-based RDTs should be able to detect parasites on the first day of symptoms, and that the persistence of the antigen will cause the tests to remain positive for at least seven days after treatment. The duration of a positive test result following curative treatment is dependent on the duration and density of parasitaemia prior to treatment and the presence and affinity of antiPfHRP2 antibodies.

Keywords: Histidine-rich protein, Rapid diagnostic tests, Plasmodium falciparum

\section{Background}

Plasmodium falciparum causes the most severe form of malaria, and contributes to high morbidity and mortality in areas of the world where malaria is endemic. Accurate diagnostics are paramount for efficient treatment of falciparum malaria. In this context, the popularity of malaria rapid diagnostic tests (RDTs) has increased due

\footnotetext{
* Correspondence: michelle.gatton@qimr.edu.au

${ }^{4}$ Malaria Drug Resistance and Chemotherapy Laboratory, Queensland Institute of Medical Research, Locked Bag 2000, Royal Brisbane Hospital, HerstonQld 4029, Australia

Full list of author information is available at the end of the article
}

to the WHO recommendation for parasitological confirmation of suspected malaria cases prior to treatment [1]. Compared to microscopy, RDTs are easier to use, do not require well-maintained microscopes and skilled microscopists, and provide a timely result. The majority of $P$. falciparum specific RDTs function by detecting a water soluble protein produced by $P$. falciparum, namely histidine-rich protein 2 ( $P f \mathrm{HRP} 2$ ). Detection is accomplished using monoclonal antibodies specific for PfHRP2. PfHRP2 is produced by the parasite throughout its asexual lifecycle; it is expressed on the surface of infected erythrocytes and released into the peripheral 
circulation during schizogony [2,3]. Due to the persistence of the protein in the circulation, PfHRP2-detecting RDTs have however been reported to have low specificity for diagnosis of current malaria infection in areas of high transmission [4] and following treatment [5,6]; that is, the PfHRP2-detecting RDTs may return positive results when parasites are not present in the blood. This persistence also affects the suitability of PfHRP2-detecting RDTs for diagnosis of malaria in endemic regions where chronic asymptomatic infections are common. In addition, the timing of onset of detectable antigenaemia using the RDT, relative to the appearance of parasites in the bloodstream at a level detectable by standard microscopic examination of a thick blood film is not well defined.

As mature $P$. falciparum parasites sequester in splanchnic vascular beds during the last half of the asexual life-cycle they are not accessible for microscopic diagnosis at this time. An additional consequence of sequestration is that the total parasite biomass may be underestimated if peripheral blood sampling is relied upon. It has, therefore, been proposed that quantitative assays of PfHRP2 level provide a more accurate measurement of parasite biomass and potentially assist in determining the prognosis of severe malaria $[2,7]$.

The kinetics of PfHRP2 antigenaemia are not well understood, although key parameters such as rate of production and elimination half-life have been estimated from in vitro and in vivo studies $[7,8]$. In the current study a model of the kinetics of PfHRP2 is developed and used to assess its potential impact on the interpretation of malaria RDT results. This model was applied to clinical data to estimate circulating PfHRP2 levels, as well as the amount of PfHRP2 available for detection using RDTs that include a red blood cell (RBC) lysis step.

\section{Methods}

\section{Modelling PfHRP2 kinetics}

A mathematical model was developed to mimic the kinetics of PfHRP2, incorporating both the production and clearance of the protein during a $P$. falciparum infection. In developing the model it was assumed that the host was infected with a single, relatively synchronous infection of $P$. falciparum with a two-day (48 hour) asexual life cycle.

Studies of in vitro culture of $P$. falciparum indicate that PfHRP2 accumulates in the cell as the parasite develops within the erythrocyte with little membrane leakage, resulting in 78-99\% of PfHRP2 being released into the circulation when the erythrocyte ruptures during schizogony [2]. The model assumed production of PfHPR2 occurred early in the asexual cycle, with all the protein remaining within the infected $R B C$ (iRBC) until the time of schizont rupture when it is instantaneously released into the circulation. The number of parasites replicating (i.e. schizonts rupturing) at a given time during an infection was estimated from available clinical data. Where required, parasite density was converted to absolute number of parasites by multiplying the density by an assumed blood volume of $5 \mathrm{~L}\left(5 \times 10^{6} \mu \mathrm{L}\right)$.

Two scenarios for PfHRP2 distribution within the body were considered; 1 ) the minimum circulating concentration, estimated assuming that PfHRP2 is watersoluble and distributed in equilibrium throughout the extracellular water volume of 14 L [9], 2) maximum circulating concentration, estimated assuming PfHPR2 is distributed only within the total blood volume of $5 \mathrm{~L}$.

The elimination of PfHRP2 from the circulation was assumed to be continuous following a first-order decay process:

$$
d_{k}=\exp \left[\frac{\ln (0.5) k}{3.67}\right]=\exp [-0.18887 k]
$$

where $d_{k}$ is the proportion of PfHRP2 remaining $k$ days after it was produced $(k=0, \ldots, 25)$. The exponent was based on the published PfHRP2 half-life of 3.67 days [7].

The absolute amount of PfHRP2 (in grams) for any day during an infection $\left(H_{t}\right)$ was calculated using equation (2), while the minimum $\left(\operatorname{Hmin}_{t}\right)$ and maximum $\left(\right.$ Hmax $\left._{t}\right)$ circulating concentrations of PfHRP2 $(\mathrm{g} / \mu \mathrm{L})$ were calculated according to equations (3) and (4), respectively.

$$
H_{t}=f \sum_{j=1}^{t} d_{t-j} p_{j}
$$

where $t$ is the time in days with $t=1$ being the first schizogony of the parasite, $p_{j}$ is the number of parasites within the host replicating on day $j$, and $f$ is the amount of PfHRP2 produced per iRBC (g).

$$
\begin{aligned}
& \operatorname{Hmin}_{t}=\frac{H_{t}}{14 \times 10^{6}} \\
& \operatorname{Hmax}_{t}=\frac{H_{t}}{5 \times 10^{6}}
\end{aligned}
$$

The concentration of PfHRP2 measured by RDT or ELISA $\left(\operatorname{Tmin}_{t}\right.$ and $\operatorname{Tmax}_{t}$ ) exceeds $\mathrm{Hmin}_{t}$ and $\operatorname{Hmax}_{t}$ since it contains the circulating PfHRP2 plus the PfHRP2 within the iRBCs which is released due to cell lysis as a consequence of an RDT or ELISA assay.

$$
\operatorname{Tmin}_{t}=\operatorname{Hmin}_{t}+\frac{f q_{t}}{5 \times 10^{6}}
$$




$$
\operatorname{Tmax}_{t}=\operatorname{Hmax}_{t}+\frac{f q_{t}}{5 \times 10^{6}}
$$

where $q_{t}$ is the number of parasites within the host on day $t\left(q_{t}=p_{t}\right.$ on replication days in a synchronous infection).

\section{Study datasets}

To fit the model and assess sensitivity, the PfHRP2 kinetics model was applied to two datasets.

Study 1. The first dataset was from a prospective, unblinded, Phase IIa clinical trial where volunteers were infected with blood stage P. falciparum [10]. The subjects were infected with approximately 1,800 P. falciparum (3D7 strain) asexual parasites, and were given curative treatment soon after reaching the target parasitaemia of $\geq 1,000$ parasites $/ \mathrm{mL}$, as determined by PCR quantification [11]. Details of the study, including ethics approval have been previously reported [10]. For the current study data from subjects in the third study cohort were considered, and of the nine subjects in this cohort, three (subjects 12, 13 and 14) had sufficient numbers of pre-treatment data points to calibrate the model. Parasite density and corresponding PfHRP2 concentration were estimated from blood samples taken at pre-specified time points (approximately once or twice daily) from initial infection to parasite clearance.

To determine the concentration of circulating PfHRP2, serial blood samples from each subject were assessed by PfHRP2 ELISA (Malaria Ag. Pf. ELISA Standard Diagnostics Korea; product code 05EK50). To interpolate the amount of PfHRP2 present in the blood samples, a calibration curve was constructed using serial dilutions of a 3D7 P. falciparumculture supernatant and used as control standards in each ELISA. The concentration of PfHRP2 in this culture supernatant had previously been measured at $55.5 \mathrm{ng} / \mathrm{mL}$ by interpolating the ELISA optical density of serial dilutions against a stock of recombinant PfHRP2 protein with known concentration (Lee, personal communication). PfHRP2 concentration within each sample was determined by interpolating the optical density of the sample with the standard curve using the software package Softmax Pro (Molecular Devices Inc.).

The pre-treatment parasitaemia data was used to fit the $f$ parameter in the maximum concentration model. As the PfHRP2 quantification was conducted using RBC pellets, rather than the serum, only the maximum concentration model was considered during the model fitting. An initial value of $f\left(f_{0}\right)$ was assumed to be $5.2 \times$ $10^{-15} \mathrm{~g}$, as this was the median value previously reported for four $P$. falciparum isolates during in vitro culture [8]. Calibration of the model to calculate the optimal value for $f\left(f^{*}\right)$ was achieved by determining the multiplication factor $(m)$ which produced the minimum residual sum of squares between the predicted PfHRP2 concentration and subject ELISA data such that $f^{*}=$ $f_{0} m$.

Study 2. The second dataset was a sample of three patients with neurosyphilis who had been treated by infection with P. falciparum [12]. Patients S561, S707 and S811 were selected as illustrative examples of a 'natural infection', as the malaria infection was not treated with antimalarial drugs to modify the primary attack. Patients S561 and S811 had no previous history of malaria infection and were infected with the McLendon strain of $P$. falciparum (blood-induced), whilst Patient S707 was reinfected with the McLendon strain of P. falciparum after being infected with the same strain 30 days prior [13]. The parasitaemia data from this study set was used to investigate the kinetics of PfHRP2 antigenaemia in symptomatic, untreated infections.

\section{Threshold of detection of malaria RDTs}

To assess the minimum PfHRP2 concentration detectable by malaria RDTs four different RDT products were tested against serial dilutions of 3D7 parasite culture supernatant containing a known concentration of PfHRP2. This supernatant was the same used to generate the standard curve in the ELISA assay described for Study 1 above. The products tested were Firstsign ${ }^{\mathrm{TM}}$ ParaView (Pan + Pf) Malaria Test (Catalogue \# 2101 CB-25, Unimed International Inc), SD BIOLINE Malaria Ag Pf/Pv (Catalogue \# 05FK80, Standard Diagnostics Inc), Carestart ${ }^{\mathrm{TM}}$ Malaria HRP2/pLDH (Pf/PAN) COMBO (Catalogue \# G0131, AccessBioInc) and ICT Malaria Combo Cassette Test (Catalogue \#ML02, ICT Diagnostics).

Each sample with a specified concentration of PfHRP2 was added to the RDTs as per the manufacturer's instructions with the test scored as positive if the PfHRP2 band was visible and negative if there was no visible PfHRP2 band. The reader of the RDT was not blinded to the concentration of PfHRP2 added to the RDT. The maximum dilution producing a positive result was classified as the RDT detection threshold, and the concentration of PfHRP2 corresponding to this dilution was calculated.

\section{Results}

Estimation of amount of PfHRP2 produced by parasites The PfHRP2 model was calibrated using data from Study 1 . The optimal individualized multiplication factor ( $m$ ) for subjects varied between 17.5 and 39.4, suggesting that the amount of PfHRP2 produced by the 3D7 $P$. falciparum parasites was considerably higher than previously reported (Table 1 ). The mean multiplication factor for the three subjects was 27.1 , resulting in an 
Table 1 Optimal multiplication factor for production of PfHRP2 by 3D7 P.falciparum parasites

\begin{tabular}{cccccc}
\hline Subject & Treatment day & No. pre-treatment data points & Optimal multiplication factor $(\boldsymbol{m})$ & Estimated $P f H R P 2$ produced/parasite $\left(\boldsymbol{f}^{*}\right)$ \\
\hline 12 & 7.5 & 4 & 24.4 & $127 \mathrm{fg}$ \\
\hline 13 & 7.5 & 3 & 17.5 & $91 \mathrm{fg}$ \\
\hline 14 & 9.5 & 6 & 39.4 & $205 \mathrm{fg}$ \\
\hline
\end{tabular}

The estimated quantity of PfHRP2 produced per parasite is calculated as $m \times 5.2 \mathrm{fg}$.

estimate of $1.4 \times 10^{-13} \mathrm{~g}$ of PfHRP2 produced per parasite per replication cycle. The fit of the predicted $P f H R P 2$ quantities for each individual using the mean optimal multiplication factor is presented in Figure 1.

\section{Kinetics of PfHRP2 in untreated infections}

The model was used to investigate the kinetics of PfHRP2 antigenaemia during an infection by using parasitaemia data from the three neurosyphilis patients (S561, S707 and S811) in Study 2. The amount of circulating PfHRP2 (Hmin ${ }_{t}$ and $\operatorname{Hmax}_{t}$ ), as well as the amount of PfHRP2 predicted to be measurable by ELISA $\left(\operatorname{Tmin}_{t}\right.$ and $\operatorname{Tmax}_{t}$ ) were calculated (Figure 2).

The duration and first day that PfHRP2 could be detected at concentrations between $10 \mathrm{ng} / \mathrm{mL}$ and 100 $\mathrm{ng} / \mathrm{mL}$ were estimated for each patient. Due to the potential for different $P$. falciparum parasite strains to produce different quantities of PfHRP2 $(f)$, the model was applied using the optimal value determined in Study 1 (fitted model, $f=1.4 \times 10^{-13} \mathrm{~g}$ ) as well as the previously reported value of $5.2 \times 10^{-15} \mathrm{~g}$ [8] (unadjusted model) (Table 2). Considering a threshold of 100 $\mathrm{ng} / \mathrm{mL}$, the first day that PfHRP2 was predicted to be detectable was Day 2 for all three patients under the fitted model, and Day 4 or Day 7 under the unadjusted model for patients S561 and S811, and S707, respectively. For the fitted model, the number of days that PfHRP2 was predicted to be greater than $100 \mathrm{ng} / \mathrm{mL}$ ranged between 25 and 50 days under the minimum circulation model and between 29 and 56 days under the maximum circulation model. The duration during an infection when the PfHRP2 antigenaemia was above the nominated thresholds was always longer for the maximum circulation model compared to the minimum circulation model, and the model output was highly sensitive to changes in $f$. The fitted model always produced a longer period of antigenaemia above a threshold, and an equal or earlier transition to this state, than the unadjusted model, due to the higher production of PfHRP2 per parasite per replication cycle.

The parasitaemia time-course from subjects in Study 1 was used to assess the longevity of circulating PfHRP2 following curative treatment. In these three subjects the amount of PfHRP2 decayed to be less than $110 \mathrm{ng} / \mathrm{L} 12$
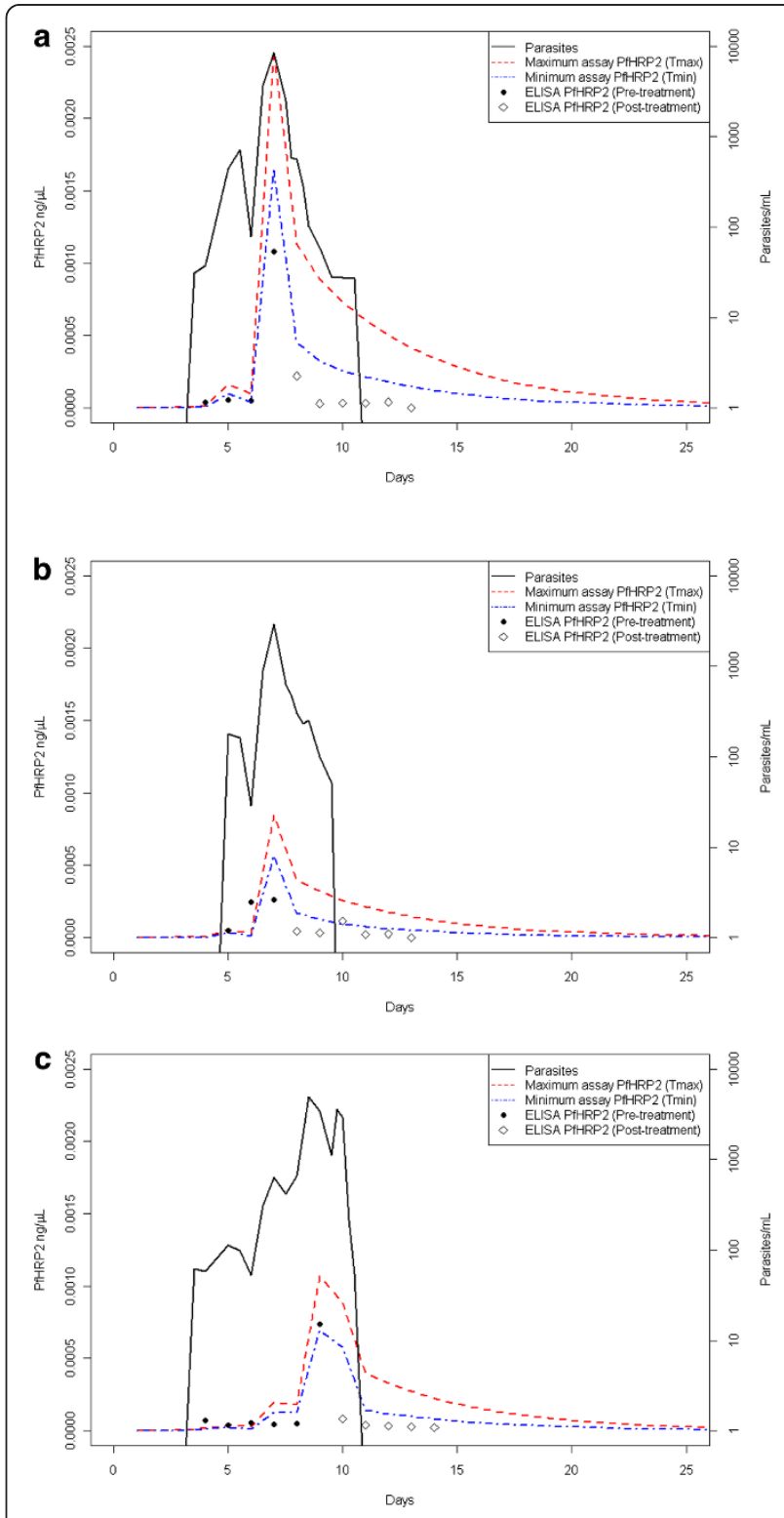

Figure 1 Parasitaemia and ELISA data from Study 1, along with the predictedPfHRP2 concentration. Data are presented for a) Patient 12, b) Patient 13 and c) Patient 14. The PfHRP2 concentrations were predicted by applying the fitted model with the mean optimal PfHRP2 production of $1.4 \times 10^{-13} \mathrm{~g}$ per parasite per replication cycle. 

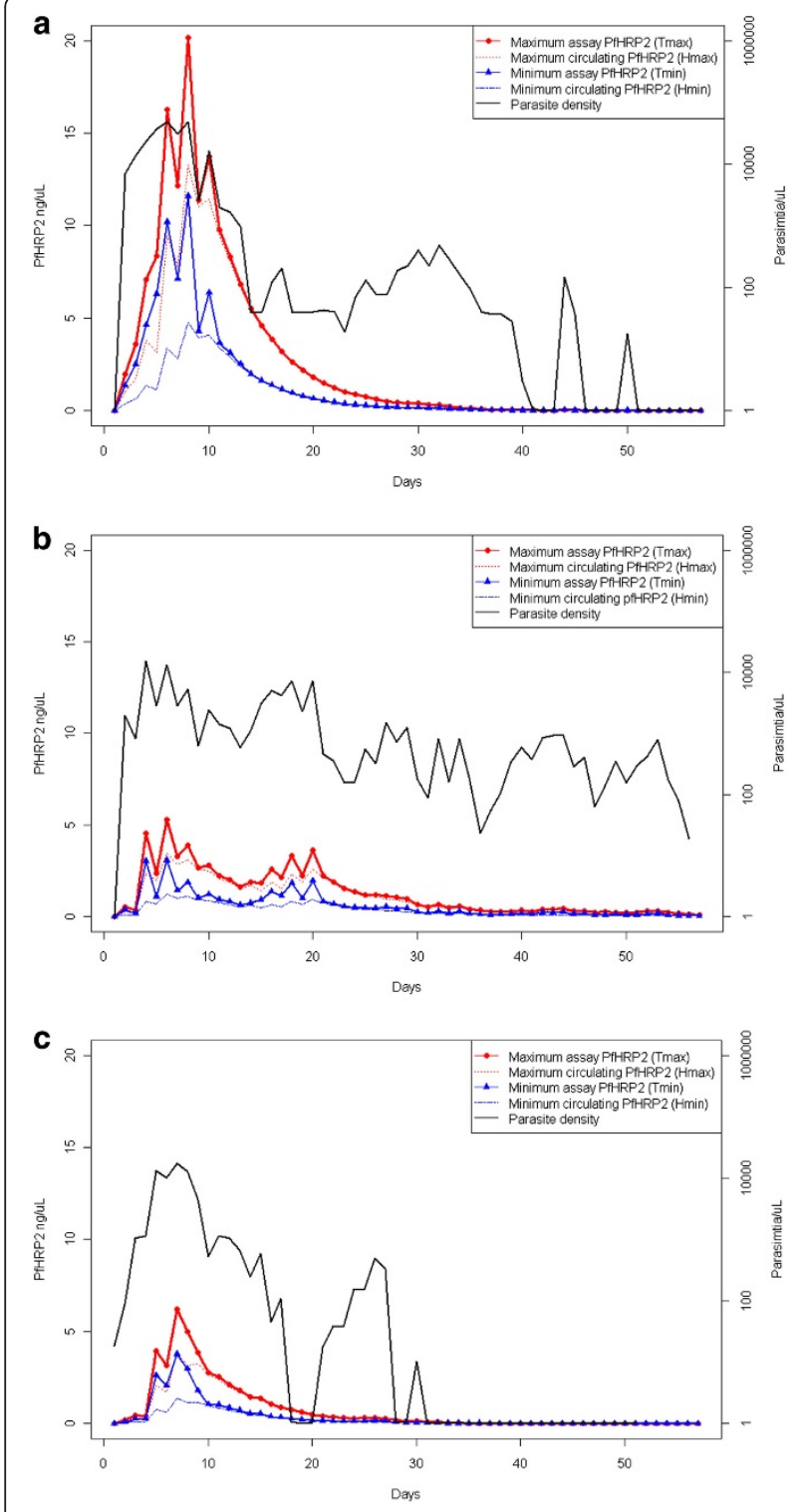

Figure 2 Parasitaemia and estimatedPfHRP2 concentrations over the course of untreated infections. Data are presented for patients a) S561, b) S707 and c) S811 in Study 2. Predicted PfHRP2 concentrations are for the maximum $\left(H_{\max }\right)$ and minimum $\left(H_{\min }\right)$ circulating PfHRP2, and the PfHRP2 concentration which would be measured during and ELISA or RDT ( $T_{\max }$ and $T_{\min }$ ) which includes intracellular PfHRP2 which is released during the assay.

days, 7 days and 8 days after curative treatment for subjects 12,13 and 14 , respectively.

\section{Chronic infections - the minimum number of parasites required to maintain PfHRP2 above a threshold}

The equilibrium parasite density required to maintain the PfHRP2 level above a nominated threshold was also determined for both the minimum and maximum circulation models (Figure 3). Applying these parasite densities resulted in the concentration of PfHRP2 gradually increasing until approximately Day 20, after which it plateaus at the equilibrium value. Linear regression models were fitted to determine the relationship between the threshold concentration $(\mathrm{ng} / \mu \mathrm{L})$ and equilibrium parasite density (parasites $/ \mu \mathrm{L}$ ). Statistically significant models were achieved $(\mathrm{P}<0.001)$ and are reported below. The values in brackets represent the SE of the coefficients.

$$
\begin{aligned}
& \text { Equilbrium no. of parasites }(\text { minimum model })=0.7+2311.9 \times \text { PfHRP2 threshold } \\
& \text { (0.1) (2.3) }
\end{aligned}
$$

These estimates of the equilibrium number of parasites represent the minimum number of parasites required consistently over time to maintain the level of PfHRP2 above a nominated threshold. Parasite densities above the equilibrium would always produce PfHRP2 levels over the threshold. Fluctuating parasite densities, as may be seen in chronic infections, will also maintain PfHRP2 levels above the threshold provided the period of parasitaemia below the equilibrium value is short; the maximum duration of low parasitaemia will be dependent on the parasite dynamics and amount of PfHRP2 produced immediately prior to the drop in parasite density.

\section{Malaria RDT detection thresholds}

The performances of each RDT against known PfHRP2 concentrations are shown in Table 3. The lowest dilutions at which positive test results occurred were 27.75 $\mathrm{ng} / \mathrm{mL}, 6.94 \mathrm{ng} / \mathrm{mL}$ and $6.94 \mathrm{ng} / \mathrm{mL}$ for the Firstsign ${ }^{\mathrm{TM}}$ ParaView (Pan + Pf) Malaria Test, SD BIOLINE Malaria Ag Pf/Pv, and Carestart ${ }^{\mathrm{TM}}$ Malaria HRP2/pLDH (Pf/ PAN) COMBO (Catalogue \# G0131, AccessBioInc), respectively. The ICT Malaria Combo Cassette Test produced a faint positive band when tested against the negative control (culture media) and the lowest PfHRP2 dilutions (Table 3 ). The band intensity for this product increased when tested with a sample containing 13.88 $\mathrm{ng} / \mathrm{mL}$ of PfHRP2, so this concentration was considered to be the minimum level of detection for this product.

Applying these detection thresholds to the estimated equilibrium equations above indicates that the threshold parasitaemia that would be required to maintain a positive RDT in a chronic infection would be between 8 and 65 parasites $/ \mu \mathrm{L}$, dependent on the distribution of PfHRP2 within the body and the specific product (Table 4).

\section{Discussion}

Understanding the kinetics of PfHRP2 is important to understanding the performance characteristics of 
Table 2 Characteristics of predicted PfHRP2 kinetics in illustrative untreated infections

\begin{tabular}{|c|c|c|c|c|c|c|}
\hline \multirow[t]{2}{*}{ Patient } & \multirow{2}{*}{$\begin{array}{l}\text { Day of } \\
\text { first fever }\end{array}$} & \multirow{2}{*}{$\begin{array}{l}\text { PfHRP2 } \\
\text { threshold (ng/ } \\
\mathrm{mL} \text { ) }\end{array}$} & \multicolumn{2}{|c|}{ Fitted model } & \multicolumn{2}{|c|}{ Unadjusted model } \\
\hline & & & $\begin{array}{l}\text { First day above threshold } \\
\text { (MinCM, MaxCM) }\end{array}$ & $\begin{array}{c}\text { Days above } \\
\text { threshold (range) }\end{array}$ & $\begin{array}{l}\text { First day above threshold } \\
\text { (MinCM, MaxCM) }\end{array}$ & $\begin{array}{c}\text { Days above } \\
\text { threshold (range) }\end{array}$ \\
\hline \multirow[t]{3}{*}{ S561 } & 2 & 100 & 2,2 & $32-34$ & 4,3 & $9-15$ \\
\hline & & 20 & 2,2 & $39-46$ & 2,2 & $19-25$ \\
\hline & & 10 & 2,2 & $44-51$ & 2,2 & 24-31 \\
\hline \multirow[t]{3}{*}{5707} & 4 & 100 & 2,2 & $25-29$ & 7,5 & $2-6$ \\
\hline & & 20 & 2,2 & $31-35$ & 5,5 & $10-15$ \\
\hline & & 10 & 2,1 & $34-40$ & 5,3 & $13-25$ \\
\hline \multirow[t]{3}{*}{5811} & 2 & 100 & 2,2 & $50-56$ & 4,4 & $2-7$ \\
\hline & & 20 & 2,2 & $59-64$ & 4,2 & $21-31$ \\
\hline & & 10 & 2,2 & $63-68$ & 2,2 & $29-47$ \\
\hline
\end{tabular}

The kinetics are summarized by the day they first exceed a nominated threshold under the minimum (MinCM) and maximum circulating PfHRP2 models (MaxCM) and the total number of days they are above this threshold. A range for the number of days above the threshold is given by combining the results from the MinCM and MaxCM models. Results are presented using the mean PfHRP2 production fitted in this study (fitted model) and also the previously reported PfHRP2 production of $5.2 \mathrm{fg} /$ parasite/replication cycle (unadjusted model) [8].

malaria RDTs that detect this antigen. For optimal clinical diagnosis RDTs need to return a positive result at the time of first fever in malaria naïve individuals. In contrast, the slow decay of the antigen has implications for the utility of PfHRP2-based RDTs in endemic countries where low level infections are prevalent, and for the detection of recrudescent infections following treatment $[5,6]$.

A key parameter in modelling the kinetics of PfHRP2 antigenaemia is the amount of $P f \mathrm{HRP} 2$ produced by one parasite during one replication cycle $(f)$. It has been

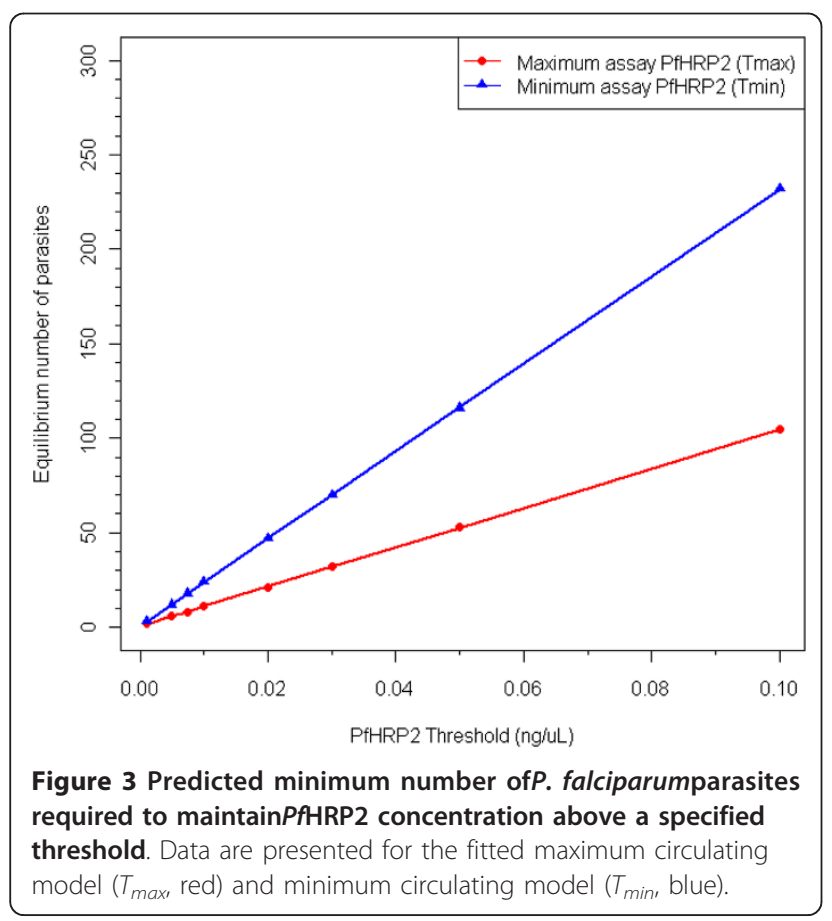

previously reported that each $P$. falciparum parasite produces $5.2 \mathrm{fg}$ (range, $1.1-13.0 \mathrm{fg}$ ) of PfHRP2 per cycle [8]. However, when this production level was used in the model, the concentration of PfHRP2 was predicted to be considerably lower than that actually measured in malaria naïve individuals infected with blood-stage 3D7 $P$. falciparum parasites. This difference may be a consequence of the differences between testing antigen production from cultures grown in vitro compared to in vivo measurements, a consequence of measurement error and stochastic variability, or the result of natural variation in protein production between different parasite strains [14]. The subjects in Study 1 were all infected by the same 3D7 parasite stain so genetic variability in the production of PfHRP2 should be minimal. Therefore, the two-fold variation between the calculated optimal values for the amount of PfHRP2 produced per parasite per replication cycle is likely a result of measurement error in the low parasite densities or ELISA assay, or stochastic variability in PfHRP2 production by individual parasites, possibly in response to host factors.

The model developed was applied under two assumptions: 1) a minimum concentration model in which the PfHRP2 was assumed to be distributed throughout the extracellular water of the host and 2) a maximum concentration model in which the PfHRP2 was assumed to be restricted to the bloodstream only. It is highly likely that the actual distribution of PfHRP2 is somewhere between these two extremes so the values presented should be viewed in this context. For simplicity, it was assumed in the model that PfHRP2 was produced early in the asexual life cycle, although in vitro data indicate that PfHRP2 accumulates throughout the cycle [2]. This assumption will not impact the calculations for the 
Table 3 Malaria RDT reactivity againstP.falciparumculture supernatant

\begin{tabular}{|c|c|c|c|c|c|c|c|}
\hline \multirow[t]{2}{*}{ Product } & \multicolumn{7}{|c|}{ Intensity of PfHRP2 band } \\
\hline & $\begin{array}{c}27.75 \mathrm{ng} / \\
\mathrm{mL}\end{array}$ & $\begin{array}{c}13.88 \mathrm{ng} / \\
\mathrm{mL}\end{array}$ & $\begin{array}{c}6.94 \mathrm{ng} / \\
\mathrm{mL}\end{array}$ & $\begin{array}{c}3.47 \mathrm{ng} / \\
\mathrm{mL}\end{array}$ & $\begin{array}{c}1.73 \mathrm{ng} / \\
\mathrm{mL}\end{array}$ & $\begin{array}{c}0.87 \mathrm{ng} / \\
\mathrm{mL}\end{array}$ & $\begin{array}{l}0 \mathrm{ng} / \mathrm{mL} \text { (-ve } \\
\text { control) }\end{array}$ \\
\hline $\begin{array}{l}\text { Firstsign }{ }^{\mathbf{T M}} \text { - ParaView (Pan + Pf) Malaria } \\
\text { Test }\end{array}$ & 1 & Neg & Neg & Neg & Neg & Neg & Neg \\
\hline SD BIOLINE Malaria Ag Pf/Pv & 4 & 2 & 1 & Neg & Neg & Neg & Neg \\
\hline CarestartTM Malaria HRP2/pLDH & 1 & 1 & 1 & Neg & Neg & Neg & Neg \\
\hline ICT Malaria Combo Cassette Test & 4 & 2 & 1 & 1 & 1 & 1 & 1 \\
\hline
\end{tabular}

Table entries are the intensities of the PfHRP2 band, scored from 1 (faint band) to 4 (strong band). Neg indicates no PfHRP2 band was visible.

amount of circulating antigen, but may produce a slight overestimation of the amount of antigen measurable by RDT or ELISA. The magnitude of the overestimation will depend on the relative amount of antigen contained within the cells at the time of the assay compared to the amount of circulating antigen, factors dependent on the parasite density and duration of the infection prior to sampling.

It was recently reported that a commercial PfHRP2 ELISA kit could detect PfHRP2 concentrations down to $110 \mathrm{ng} / \mathrm{L}$ [15]. In the current study where four commercial RDTs were tested against the same PfHRP2 antigen, the diagnostic sensitivity of the tests varied between 6.9 $\mu \mathrm{g} / \mathrm{L}$ and $27.7 \mu \mathrm{g} / \mathrm{L}$ of PfHRP2. The model output shows that for the fitted model, these values are achieved on, or immediately prior, to the first day of fever for the three patients in Study 2. In Study 1, the predicted amount of PfHRP2 would have been insufficient to produce a positive RDT test, as subjects were treated before becoming symptomatic, with peak parasite densities less than 10 parasites $/ \mu \mathrm{L}$. Field data indicates that PfHRP2-detecting RDTs can detect parasite densities less than 100 parasites/ $\mu \mathrm{L}[16,17]$. The model predictions indicate that the malaria RDTs tested here would be able to diagnose an infection at the time of first fever. A lower level of PfHRP2 production as in the unadjusted model, or higher detection thresholds in the RDT may cause a delay in a positive RDT result.

The model informs understanding on how duration of infection and parasite density impact on the longevity of PfHRP2 in natural infections. This replicates field data, where children with higher parasite densities at the time of treatment had a significantly longer antigen persistence compared to children with lower parasite densities [18]. In Study 1, subjects were treated prior to becoming symptomatic, and the peak parasitaemia was much lower than would occur in a natural infection. Thus, the longevity of PfHRP2 in this circumstance is a likely underestimate of the expected duration of a detectable antigenaemia in symptomatic infection. Indeed, as few as eight parasites/ $\mu \mathrm{L}$ may be required to maintain PfHRP2 equilibrium above $6.9 \mu \mathrm{g} / \mathrm{L}$ using the parameter space described.

As has been reported before, the diagnostic sensitivity of malaria RDTs targeting PfHRP2 is varied. The four products examined here have also been assessed as part of the WHO Malaria Rapid Diagnostic Test Programme where they were tested against wild-type isolates $[19,20]$. Each product performed well when tested against $200 P$. falciparum parasites/ $\mu \mathrm{L}$, with overall positivity rates varying from $93.0 \%$ for Firstsign ${ }^{\mathrm{TM}}$ - ParaView (Pan + Pf) Malaria Test to $99.1 \%$ for Carestart ${ }^{\mathrm{TM}}$ Malaria HRP2/pLDH (Pf/PAN) COMBO. The results from this study are generally aligned with the product testing results, as the product with the lowest panel detection score, Firstsign ${ }^{\mathrm{TM}}$ - ParaView (Pan + Pf) Malaria Test, had the highest PfHRP2 detection threshold. The modelling results indicate that these tests should continue to perform consistently well at concentrations in the order of 50-100 parasites $/ \mu \mathrm{L}$, or better in some cases. This result reflects a previous study in which the ICT Malaria Combo Cassette Test was able to detect $P$. falciparum

Table 4 Predicted equilibrium parasite densities required to maintain a positive RDT test

\begin{tabular}{|c|c|c|c|}
\hline Product & $\begin{array}{l}\text { Min. detectable PfHRP2 conc. } \\
\text { (ng/mL) }\end{array}$ & $\begin{array}{l}\text { Est. no. parasites for MinCM } \\
\text { (parasites } / \mu \mathrm{L} \text { ) }\end{array}$ & $\begin{array}{l}\text { Est. no. parasites for MaxCM } \\
\text { (parasites/ } / \mathrm{L} \text { ) }\end{array}$ \\
\hline $\begin{array}{l}\text { Firstsign } \\
\text { Malaria - ParaView }(\text { Pan }+ \text { Pf })\end{array}$ & 27.75 & 64.9 & 29.6 \\
\hline 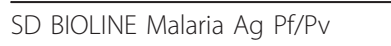 & 6.94 & 16.7 & 7.8 \\
\hline Carestart $^{\mathrm{TM}}$ Malaria HRP2/pLDH & 6.94 & 16.7 & 7.8 \\
\hline ICT Malaria Combo Cassette Test & $13.88^{*}$ & 32.8 & 15.1 \\
\hline
\end{tabular}

* The lowest dilution which gives a band intensity $>1$ to adjust for false positive result on negative control.

MinCM and MaxCM represent the minimum circulating model and maximum circulating model, respectively. 
parasites below $100 / \mu \mathrm{L}$ in field samples [17]. However it is important to note that the four products tested in the current study were within the top $40 \%$ of RDTs tested by the WHO programme, as ranked by their performance against wild-type $P$. falciparum isolates at 200 parasites $/ \mu \mathrm{L}[21]$, and in addition were stored in ideal conditions. Therefore, extrapolation of the results obtained here to other RDTs is not recommended, particularly those products not achieving the same level of performance as the tested sub-set.

The model and results presented in the current study assume all PfHRP2 antigen produced by parasites in natural human infection is available for measurement. However, it is possible that PfHRP2 binds or complexes with other ligands or proteins such as PfHRP2-specific antibodies, thereby reducing the amount available as free circulating antigen. In this respect, it is known that anti-PfHRP2 antibodies develop and may thereby act to reduce the amount of free PfHRP2 circulating [3]. While these factors have not been considered in the current model, it is likely their effect would primarily influence the longevity of the antigen in the circulation after treatment. As RDTs cause RBC lysis, while there is an active infection and parasites within the host, a blood sample tested using an RDT would be able to detect the PfHRP2 contained within the iRBCs provided that the quantity of antibody in the serum is insufficient to bind all of the PfHRP2 released from the iRBCs before it reacts with the test band in the RDT. A caveat to this is that the parasites must be present in the blood sample tested by the RDT. Parasite sequestration can affect the availability of parasites in finger-prick samples, a factor which has not been considered in this model.

\section{Conclusion}

Malaria RDTs are becoming the cornerstone of effective malaria diagnosis in primary healthcare settings. Understanding the benefits and limitations of these tools is important to guide clinicians and product development. The outputs of the mathematical model reported here indicate that the PfHRP2-based RDTs that were tested should be able to detect parasites on the first day of symptoms, and that the persistence of the antigen will cause the tests to remain positive for at least seven days post-treatment. The post-treatment duration of positive tests is dependent on the duration and density of parasitaemia prior to treatment, and possibility other factors such as anti-PfHRP2 antibodies. In chronic infections a steady state parasitaemia of only 4-65 parasites $/ \mu \mathrm{L}$ is likely to be sufficient to maintain a positive RDT result.

\section{Acknowledgements}

The clinical trial that provide the Study 1 data was funded by Medicines for Malaria. MLG is funded by a NHMRC Career Development Award and the
Research and Policy for Infectious Disease Dynamics (RAPIDD) program of the Science and Technology Directorate, Department of Homeland Security, and the Fogarty International Center, National Institutes of Health. JMC was supported by a NHMRC Practitioner Fellowship, and a Government of Queensland Clinical Research Fellowship.

\section{Author details}

${ }^{1}$ Statistics Unit, Queensland Institute of Medical Research, Herston, Australia. ${ }^{2}$ Clinical Tropical Medicine Laboratory, Queensland Institute of Medical Research, Herston, Australia. ${ }^{3}$ School of Medicine, University of Queensland, Herston, Australia. ${ }^{4}$ Malaria Drug Resistance and Chemotherapy Laboratory, Queensland Institute of Medical Research, Locked Bag 2000, Royal Brisbane Hospital, HerstonQld 4029, Australia.

\section{Authors' contributions}

LM developed the model, conducted the analysis and drafted the manuscript. AB performed the laboratory testing of RDTs and ELISA assays. JMC participated in the design of the study. MLG participated in the design of the study, reviewed the model output and helped draft the manuscript. All authors read and approved the final manuscript.

\section{Competing interests}

The authors declare that they have no competing interests.

Received: 22 December 2011 Accepted: 19 March 2012 Published: 19 March 2012

\section{References}

1. World Health Organization: Guidelines for the treatment of malaria. Geneva, 22010.

2. Desakorn V, Silamut K, Angus B, Sahassananda D, Chotivanich K, Suntharasamai P, Simpson J, White NJ: Semi-quantitative measurement of Plasmodium falciparum antigen PfHRP2 in blood and plasma. Trans $R$ Soc Trop Med Hyg 1997, 91:479-483.

3. Biswas S, Tomar D, Rao DN: Investigation of the kinetics of histidine-rich protein 2 and of the antibody responses to this antigen, in a group of malaria patients from India. Ann Trop Med Parasitol 2005, 99:553-562.

4. Abeku TA, Kristan M, Jones C, Beard J, Mueller DH, Okia M, Rapuoda B, Greenwood B, Cox J: Determinants of the accuracy of rapid diagnostic tests in malaria case management: evidence from low and moderate transmission settings in the East African highlands. Malar J 2008, 7:202.

5. Houze S, Boly MD, Le Bras J, Deloron P, Faucher JF: PfHRP2 and PfLDH antigen detection for monitoring the efficacy of artemisinin-based combination therapy (ACT) in the treatment of uncomplicated falciparum malaria. Malar J 2009, 8:211.

6. Mayxay M, Pukrittayakamee S, Chotivanich K, Looareesuwan S, White NJ: Persistence of Plasmodium falciparum HRP-2 in successfully treated acute falciparum malaria. Trans R Soc Trop Med Hyg 2001, 95:179-182.

7. Dondorp AM, Desakorn V, Pongtavornpinyo W, Silamut K, Chotivanich K, Newton PN, Pitisuttithum P, Smithyman AM, White NJ, Day NPJ: Estimation of the total parasite biomass in acute falciparum malaria from plasma PfHRP2. PLoS Med 2005, 2:788-797.

8. Desakorn V, Dondorp AM, Silamut K, Pongtavornpinyo W, Sahassananda D, Chotivanich K, Pitisuttithum P, Smithyman AM, Day NP, White NJ: Stagedependent production and release of histigine-rich protein 2 by Plasmodium falciparum. Trans R Soc Trop Med Hyg 2005, 99:517-524.

9. Guyton AC, Hall JE: Textbook of medical physiology. 11 edition. Pennsylvania: Elsevier Saunders; 2006.

10. McCarthy JS, Sekuloski S, Griffin PM, Elliott S, Douglas N, Peatey C, Rockett R, O'Rourke P, Marquart L, Hermsen C, Duparc S, Möhrle J, Trenholme KR, Humberstone AJ: A Pilot randomised trial of induced blood-stage Plasmodium falciparum infections in healthy volunteers for testing efficacy of new antimalarial drugs. PLOS ONE 2011, 6:e21914.

11. Rockett RJ, Tozer SJ, Peatey C, Bialasiewicz S, Whiley DM, Nissen MD, Trenholme K: McCarthy JS, Sloots TP: A real-time, quantitative PCR method using hydrolysis probes for the monitoring of Plasmodium falciparum load in experimentally infected human volunteers. Malar J 2011, 10:48.

12. Collins WE, Jeffery GM: A retrospective examination of sporozoite- and trophozoite-induced infections withPlasmodium falciparu: development of parasitologic and clinical immunity during primary infection. Am J Trop Med Hyg 1999, 61:4-19. 
13. Collins WE, Jeffery GM: A retrospective examination of secondary sporozoite and trophozoite-induced infections with Plasmodium falciparium: development of parasitologic and clinical immunity following secondary infection. Am JTrop Med Hyg 1999, 61:20-35.

14. Baker J, Gatton ML, Peters J, Ho MF, McCarthy JS, Cheng Q: Transcription and expression of Plasmodium falciparum histidine-rich proteins in different stages and strains: implications for rapid diagnostic tests. PLOS ONE 2011, 6:e22593.

15. Butterworth AS, Robertson AJ, Ho MF, Gatton ML, McCarthy JS, Trenholme KR: An improved method for undertaking limiting dilution assays for in vitro cloning of Plasmodium falciparum parasites. Malar J 2011, 10:95

16. Hendriksen IC, Mtove G, Pedro AJ, Gomes E, Silamut K, Lee SJ, Mwambuli A, Gesase S, Reyburn H, Day NP, White NJ, von Seidlein L, Dondorp AM: Evaluation of a PfHRP2 and a pLDH-based rapid diagnostic test for the diagnosis of severe malaria in 2 populations of African children. Clin Infect Dis 2011, 52:1100-1107.

17. Harris I, Sharrock WW, Bain LM, Gray KA, Bobogare A, Boaz L, Lilley K, Krause D, Vallely A, Johnson ML, Gatton ML, Shanks GD, Cheng Q: A large proportion of asymptomatic Plasmodium infections with low and submicroscopic parasite densities in the low transmission setting of Temotu Province. Solomon Islands: challenges for malaria diagnostics in an elimination setting. Malar J 2010, 9:254.

18. Kyabayinze DJ, Tibenderana JK, Odong GW, Rwakimari JB, Counihan H: Operational accuracy and comparative persistent antigenicity of HRP2 rapid diagnostic tests for Plasmodium falciparum malaria in a hyperendemic region of Uganda. Malar J 2008, 7:221.

19. World Health Organization: Malaria Rapid Diagnostic Test Performance: Results of WHO product testing of malaria RDTs: Round 1 (2008). Geneva 2009.

20. World Health Organization: Malaria Rapid Diagnostic Test Performance: Results of WHO product testing of malaria RDTs: Round 2 (2009). Geneva 2010.

21. World Health Organization: Malaria Rapid Diagnostic Test Performance: Summary results of WHO Malaria RDT Product Testing: Rounds 1-3 (20082011). Geneva 2011.

doi:10.1186/1475-2875-11-74

Cite this article as: Marquart et al: Modelling the dynamics of

Plasmodium falciparum histidine-rich protein 2 in human malaria to better understand malaria rapid diagnostic test performance. Malaria Journal 2012 11:74.

\section{Submit your next manuscript to BioMed Central and take full advantage of:}

- Convenient online submission

- Thorough peer review

- No space constraints or color figure charges

- Immediate publication on acceptance

- Inclusion in PubMed, CAS, Scopus and Google Scholar

- Research which is freely available for redistribution

Submit your manuscript at www.biomedcentral.com/submit 